\title{
Introduction for Volume 7, Issue 4
}

This issue contains 5 papers. There are 3 contributions written in English and 2 contributions in Chinese with English abstracts. The papers can be divided into three topics: financial risk, disaster reduction $\&$ emergency management, and animal infectious disease,

There are two papers in financial risk. The first paper "Research on Evaluation of Equity Financing Efficiency of Listed Companies in Strategic Emerging Industries” by Yaxi Huang \& Mu Zhang, chooses 198 listed companies in strategic emerging industries, using DEA model to study the efficiency of equity financing, and carries on efficiency analysis, investment redundancy and output shortage analysis and industry comparative analysis. The results show that the efficiency of equity financing of listed companies in strategic emerging industries is inefficient. The comprehensive efficiency, pure technical efficiency and scale efficiency are $0.370,0.603$ and 0.563 . From the scale pay, the economic scale of Chuanrungufen should be increased, Zhongguobaoan and other 179 decision-making units should be reduced; Dongxulantian and other 169 decision-making units have different levels of input redundancy and lack of output; equity financing efficiency is unevenly developed between different industries. The second paper "Wenzhou Real Estate Bubble and Rupture Risk Warning” by Anping Pan, Chongfu Huang \& Dehua Jiang, reviews the trend of housing price in Wenzhou from 2006 to 2015, and analyzes the causes of house bubble. In addition, the adjustment process of Wenzhou real estate is of typical significance, which could provide references for current market trend.

There are two papers in disaster reduction and emergency management. The first paper "Assessment of Earthquake Prevention and Disaster Reduction Capability of County-Level Administrative Units in Gansu Province” by Jin Chen, Wen Li, Wenkai Chen, et al, establishes the assessment indicator system for the earthquake prevention and disaster reduction capability of county-level administrative units. An analytic hierarchy process is applied to determine the indicator weights, and the environmental supporting capability, infrastructure supporting capability and resource supporting capability of different counties in Gansu in the handling of earthquake disasters are calculated to obtain the assessment figure for the earthquake prevention and disaster reduction capability of county-level administrative units in Gansu. The research results indicate that Anning District of Lanzhou has the strongest earthquake prevention and disaster reduction capability while Zhouqu County possesses the weakest earthquake prevention and disaster reduction capability. The second paper "Research on the Emergency Shelter Accessibility in Urban Communitie" by Bo Tang, Danni Wang, Yun Song, et al, analyzes the accessibility of emergency shelter in Yuexiu district under microcosmic perspective by Using the improved Two-Step floating catchment area method and GIS. The conclusions are as follows: Firstly, the total amount and type of emergency shelter are abundant in Yuexiu district, per capita emergency shelter is high, but spatial distribution is unreasonable; Secondly, the overall accessibility of emergency shelter is poor, north region better than south region, and supply and demand ratio is an important factor about the difference of accessibility; Thirdly, when d0 to $1500 \mathrm{~m}$, emergency shelter accessibility reaches the maximum and proportion of service area is the best. The related conclusions can provide some reference for the optimization and planning of emergency shelter in Yuexiu district.

There is one paper in disaster risk assessment, which is "Risk Assessment of Canine Distemper in the Distribution Area of Giant Panda in Sichuan, Shaanxi and Gansu Provinces, China” by Weigeng Shao, Feng Jiang,Liya Huang,et al, where the authors used MaxEnt model and combined with ArcGIS analysis to predict the potential risk of canine distemper to giant panda habitat in Sichuan, Gansu and Shaanxi. The results showed that $35.05 \%$ and $19.47 \%$ of the giant pandas were in the high risk and medium risk of canine distemper, respectively.

\section{Editors-in-Chief}

\author{
Prof. Chongfu Huang \\ Beijing Normal University \\ No.19 Xinjiekouwai Street \\ Beijing 100875, China \\ Email: hchongfu@126.com
}

\section{Prof. Gordon Huang}

Faculty of Engineering and Applied Science, University of Regina

Regina, Sask S4S 0A2, Canada

Email: gordon.huang@uregina.ca 